\title{
Oxidised cholesterol is more hypercholesterolaemic and atherogenic than non-oxidised cholesterol in hamsters
}

\author{
Chi Ho $\mathrm{Ng}^{1}$, Xiao Qiang $\mathrm{Yao}^{2}$, Yu Huang ${ }^{2}$ and Zhen-Yu Chen ${ }^{1 *}$ \\ ${ }^{1}$ Food and Nutritional Sciences Programme, Department of Biochemistry, The Chinese University of Hong Kong, Shatin, NT, \\ Hong Kong, China \\ ${ }^{2}$ Department of Physiology, The Chinese University of Hong Kong, Shatin, NT, Hong Kong, China
}

(Received 30 March 2007 - Revised 15 August 2007 - Accepted 21 August 2007)

The present study was to test the relative hypercholesterolaemic and atherogenic potency of oxidised cholesterol (OxC) and non-oxidised cholesterol in hamsters. An OxC mixture, prepared by heating pure cholesterol $(100 \mathrm{~g})$ at $160^{\circ} \mathrm{C}$ in air for $72 \mathrm{~h}$, contained $78 \%$ cholesterol and $22 \% \mathrm{OxC}$. Fifty Golden Syrian hamsters were randomly divided into five groups of ten animals and fed the control diet, a $0.05 \%$ cholesterol diet (C-0.05), a $0.10 \%$ cholesterol diet (C-0.1), a $0.05 \%$ OxC mixture diet (OxC-0.05) or a $0.10 \%$ OxC mixture diet (OxC-0.1), respectively. The OxC-0.05 and OxC-0.1 groups were more hypercholesterolaemic and had serum total cholesterol 22 and $12 \%$ higher than the corresponding C-0.05 and C-0.1 hamsters $(P<0.05)$. The OxC-0.1 group demonstrated greater deposition of cholesterol and had a larger area of atherosclerotic plaque in the aorta than the corresponding C-0.1 hamsters $(P<0 \cdot 05)$. Similarly, the aorta in the OxC-0.1 group showed greater inhibition on acetylcholine-induced relaxation compared with that in the $\mathrm{C}-0 \cdot 1$ hamsters. It was concluded that $\mathrm{OxC}$ was much more hypercholesterolaemic and atherogenic than cholesterol.

Cholesterol: Cholesterol oxidation products: Oxidised cholesterol: Oxysterol

Human diets contain both cholesterol and oxidised cholesterol $(\mathrm{OxC})$. Cholesterol is susceptible to oxidation, forming a series of cholesterol oxidation products (COP) under various food processing conditions. The amount of COP can reach up to $10 \%$ total cholesterol (TC) in foods ${ }^{1}$, particularly in Western countries where total fat intake is high and fried foods are popular ${ }^{2}$. More than thirty COP have been identified and reported ${ }^{3,4}$. The major COP include $7 \beta$-hydroxycholesterol, $7 \alpha$-hydroxycholesterol, $5 \alpha$-hydroxycholesterol, 7-ketocholesterol and $\alpha$-epoxides ${ }^{5}$.

COP have been extensively studied for their undesirable effects including cytotoxicity ${ }^{6}$, mutagenesis ${ }^{7}$ and carcinogenesis ${ }^{8}$. In addition, COP have been shown to be absorbed in a similar way to cholesterol ${ }^{9,10}$, cause disturbance of lipid metabolism $^{11}$ and alter the cell membrane function ${ }^{12,13}$. Most importantly, COP could accelerate fatty streak lesion formation and promote atherosclerosis ${ }^{14,15}$. Human daily cholesterol intake is about $300-500 \mathrm{mg}$ while that of COP could be up to $30-50 \mathrm{mg}$, provided that $10 \%$ cholesterol in the diet is oxidised. It is well known that high cholesterol consumption elevates plasma TC and LDL-cholesterol levels and increases the risk of CHD. However, the effect of COP in the diet on blood cholesterol is unfortunately ignored and there is very limited information concerning relative hypercholesterolaemic and atherosclerotic activity of $\mathrm{COP}$ and cholesterol itself $^{6}$. The present study was therefore carried out to investigate the effect of $\mathrm{OxC}$ on blood cholesterol level, atherosclerotic plaque formation and endothelium function compared with that of non-oxidised cholesterol using hamsters as an animal model.

\section{Experimental methods}

\section{Preparation of oxidised cholesterol}

Pure cholesterol was purchased from Sigma Chemical Company. GLC analysis found that COP were less than $0.1 \%$ of TC. An OxC mixture was prepared as previously described ${ }^{6}$. In brief, pure cholesterol $(100 \mathrm{~g})$ was placed into a round-bottomed flask and heated in a $160^{\circ} \mathrm{C}$ oil bath in air for $72 \mathrm{~h}$. The OxC mixture was dark-brown with an obnoxious smell. A proportion of the $\mathrm{OxC}$ mixture $(20 \mathrm{mg})$ was converted to trimethylsilyl (TMS)-ether derivatives with a Sigma TMSreagent (Sigma-Sil-A; Sigma, St Louis, MO, USA) and dissolved in the hexane. The TMS-ether derivatives were analysed in a fused silica capillary column $\left(\mathrm{SAC}^{\mathrm{TM}}-5\right.$, $30 \mathrm{~m} \times 0.25 \mathrm{~mm}$; Supelco, Inc., Bellefonte, PA, USA) in a Shimadzu GC-14B equipped with a flame ionisation detector. GLC analysis demonstrated that the OxC mixture contained $78.0 \%$ cholesterol, $8.3 \%$ 7 3 -hydroxycholesterol,

Abbreviations: C-0.1, 0.10\% cholesterol diet; C-0.05, 0.05\% cholesterol diet; COP, cholesterol oxidation product; HDL-C, HDL-cholesterol; OxC, oxidised cholesterol; OxC-0.1, 0.10\% OxC mixture diet; OxC-0.05, 0.05\% OxC mixture diet; TC, total cholesterol; TMS, trimethylsilyl.

* Corresponding author: Dr Zhen-Yu Chen, fax +852 2603 7246, email zhenyuchen@cuhk.edu.hk 
4.9\% 7-ketocholesterol, $4.6 \% 7 \alpha$-hydroxycholesterol, $2.8 \%$ $\alpha$-epoxide and $1.8 \% 5 \alpha$-hydroxycholesterol (Fig. 1).

\section{Diets}

The hypercholesterolaemic diet described by Sanders \& Sandaradura was modified and used in the present study $^{16}$. In brief, the control diet was prepared by mixing all powdered ingredients $(\mathrm{g} / \mathrm{kg})$ : maize starch, 569; casein, 200; lard, 100; sucrose, 50; mineral mix, 40; vitamin mix, 20; DL-methionine, 1. The four experimental diets were prepared by adding $0.05 \%$ non-oxidised cholesterol (C-0.05), $0.1 \%$ non-oxidised cholesterol (C-0.1), $0.05 \%$ OxC mixture (OxC-0.05) and $0.10 \%$ OxC mixture (OxC-0.1), respectively. All five powdered diets were then mixed with a gelatin solution in a ratio of $200 \mathrm{~g}$ diet per litre solution. Once the gelatin had set, the diets were cut into approximately $20 \mathrm{~g}$ cubic portions and stored frozen $\left(-20^{\circ} \mathrm{C}\right)$. It should be pointed out that the actual amount of cholesterol in the diets was greater because lard contains $0.9 \mathrm{mg}$ cholesterol $/ \mathrm{g}$, leading to an additional $0.01 \%$ cholesterol in the diets. To be more specific, TC and $\mathrm{OxC}$ accounted for $0.01,0.06,0.06,0.11$ and $0.11 \%$ in the control, C-0.05, OxC-0.05, C-0.1 and OxC-0.1 diets, respectively. All five diets contained $10 \mathrm{~g}$ lard $/ 100 \mathrm{~g}$ of the following fatty acid composition (in $\%$ of total fatty acids): myristic acid, 2.5; palmitic acid, 24.9; palmitoleic acid, 2.9; stearic acid, 19.0; oleic acid, 37.0; linoleic acid, 7.1; $\alpha$-linolenic acid, 0.4.

\section{Animals}

Fifty Golden Syrian hamsters (Mesocricetus auratus; weight $100-120 \mathrm{~g}$; age 3 months) were randomly divided into five groups $(n$ 10) and fed one of the five diets. All hamsters were housed in an animal room at $23^{\circ} \mathrm{C}$ with a $12 \mathrm{~h}$ light $-12 \mathrm{~h}$ dark cycle. To minimise the oxidation of cholesterol, the fresh diets were given to the hamsters daily, and uneaten food was discarded. Food intake was measured daily and body weight was recorded twice per week. The faeces from each hamster were pooled for a period of the 2nd, 4th and 6th weeks. At the end of 6 weeks, all

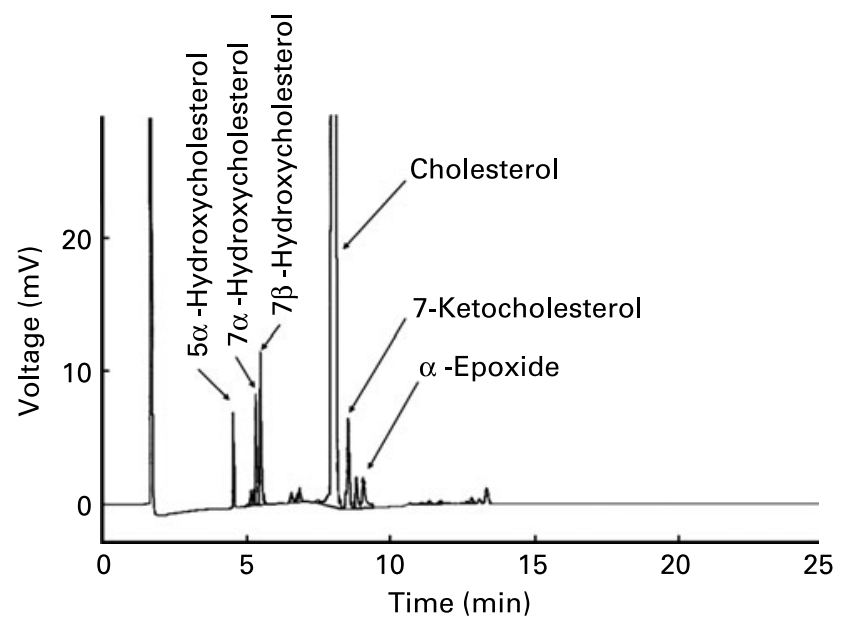

Fig. 1. Gas-liquid chromatogram of the oxidised cholesterol mixture. the hamsters were killed after overnight fasting. The blood was collected via the abdominal aorta. After clotting, the blood was centrifuged at $1500 \mathrm{~g}$ for $10 \mathrm{~min}$ and serum was collected. The liver was removed, washed with saline and stored at $-80^{\circ} \mathrm{C}$. The thoracic aorta was also removed and saved for the organ bath experiment and the analysis of atherosclerotic plaque.

\section{Determination of serum lipoproteins}

Several enzymic kits were purchased from Sigma (St Louis, MO, USA) to measure serum TAG (catalogue no. 336-20), TC (catalogue no. 352-20) and HDL-cholesterol (HDL-C; catalogue no. 352-4).

\section{Measurement of liver and aorta cholesterol}

In each case, the fresh aorta $(100 \mathrm{mg})$ or liver sample $(300 \mathrm{mg})$ was cleaned of adventitial tissue and washed in saline solution. Total lipids were extracted with the addition of $0.2 \mathrm{mg}$ stigmastanol (1.0 mg for the liver sample) as an internal standard using chloroform-methanol $(2: 1, \mathrm{v} / \mathrm{v})$. The lipid extracts were then saponified with $6 \mathrm{ml}$ of $1 \mathrm{M}-\mathrm{NaOH}$ in $90 \%$ ethanol at $90^{\circ} \mathrm{C}$ for $1 \mathrm{~h}$, and the non-saponified substances including cholesterol were converted to their TMS-ether derivatives by a commercial TMS reagent (Sigma-Sil-A; Sigma). The analysis of cholesterol TMS-ether derivatives was performed in a fused silica capillary column $\left(\mathrm{SAC}^{\mathrm{TM}}-5,30 \mathrm{~m} \times 0.25 \mathrm{~mm}\right.$ internal diameter; Supelco, Inc., Bellefonte, PA, USA) using a Shimadzu GC-14 B GLC equipped with a flame ionisation detector as previously described ${ }^{15}$.

\section{Determination of faecal neutral and acidic sterols}

Individual faecal neutral and acidic sterols were quantified as previously described ${ }^{17}$. To simplify the analysis, only faecal samples collected in week 6 were analysed because the composition of faeces was relatively constant during this period. In brief, stigmasterol $(0.3 \mathrm{mg})$ as an internal standard for neutral sterols was added to a faecal sample $(300 \mathrm{mg})$. The sample was saponified using $9 \mathrm{ml} 1 \mathrm{M}-\mathrm{NaOH}$ in $90 \%$ ethanol containing $0.3 \mathrm{mg}$ hyodeoxycholic acid as an internal standard for acidic sterols (Sigma, St Louis, MO, USA). The total neutral sterols were extracted using $8 \mathrm{ml}$ cyclohexane and were then converted to their corresponding TMS-ether derivatives for GLC analysis.

After the cyclohexane extraction, $1 \mathrm{ml} 10 \mathrm{M}-\mathrm{NaOH}$ was added to the remaining aqueous layer and heated at $120^{\circ} \mathrm{C}$ for $3 \mathrm{~h}$. After cooling down, $1 \mathrm{ml}$ of distilled water and $3 \mathrm{ml}$ $3 \mathrm{M}-\mathrm{HCl}$ were added and followed by extraction using $7 \mathrm{ml}$ diethyl ether twice. The diethyl ether layers were then pooled followed by adding $2 \mathrm{ml}$ methanol, $2 \mathrm{ml}$ dimethoxypropane and $40 \mu \mathrm{l}$ concentrated $\mathrm{HCl}(12 \mathrm{~mol} / \mathrm{l})$. After standing overnight at room temperature, the solvents were dried down and the acidic sterols were similarly converted to their TMS-ether derivatives at $60^{\circ} \mathrm{C}$ for GLC analysis.

\section{Aorta relaxation test}

Aorta relaxation was used as an indication of change in arterial functionality. In brief, the thoracic aorta from 
non-oxidised and oxidised cholesterol groups was dissected out and surrounding connective tissues and fat were removed under a dissection microscope as previously described $^{18}$. One part of the aorta was cut into $3 \mathrm{~mm}$ long aortic ring segments and each segment was suspended between two stainless-steel hooks in a $10 \mathrm{ml}$ organ bath chamber filled with normal Krebs-Henseleit solution $\left(119 \mathrm{~mm}-\mathrm{NaCl}, \quad 4.7 \mathrm{~mm}-\mathrm{KCl}, \quad 2.5 \mathrm{mM}-\mathrm{CaCl}_{2}, \quad 1 \mathrm{mM}-\mathrm{MgCl}_{2}\right.$, $25 \mathrm{~mm}-\mathrm{NaHCO}_{3}, 1.5 \mathrm{mM}-\mathrm{KH}_{2} \mathrm{PO}_{4}, 11.1 \mathrm{~mm}$-D-glucose). The chamber was bubbled with a mixture of $95 \% \mathrm{O}_{2}$ and $5 \%$ $\mathrm{CO}_{2}$ and kept at $37^{\circ} \mathrm{C}$ throughout the entire experiment. For the two stainless-steel hooks, one of them was mounted at the bottom of the bath while another one was connected to a Grass FT03 force-displacement transducer (Grass Instruments, Quincy, MA, USA) and the isometric contraction was measured by transducer and recorded by the MacLab computer system (AD Instruments, Hastings, UK). Basal tension ( $1 \mathrm{~g}$ ) was applied to all aortic rings and rings were allowed to equilibrate for $30 \mathrm{~min}$. During this time, the bath solution was replaced with pre-warmed, oxygenated Krebs-Henseleit solution three to four times. After the equilibration period, the rings were first contracted with $0.3 \mu \mathrm{M}$ phenylephrine to test vessel contractibility and then relaxed by $0 \cdot 3 \mu \mathrm{M}$-acetylcholine to assess integrity of the endothelial layer. Then, the rings were rinsed several times in prewarmed and oxygenated Krebs-Henseleit solution until the basal tension was restored and allowed to equilibrate for $60 \mathrm{~min}$. Then, the concentration-response curve to acetylcholine was constructed by adding $1 \mu \mathrm{M}$-phenylephrine and waiting until the rings' maximal contraction, followed by the addition of $3 \mathrm{nM}$ - to $10 \mu \mathrm{M}$-acetylcholine to produce a concentration-response relaxation curve to acetylcholine.

\section{Measurement of aorta atherosclerotic plaque}

The percentage area of atherosclerotic plaque on the endothelial layer was determined as previously described ${ }^{19}$. In brief, the remaining part of the thoracic aorta was cut open vertically. The aorta was then stained with $0.5 \mathrm{~g}$ Sudan III in $10 \mathrm{ml}$ ethanol for $3 \mathrm{~h}$. The endothelial layer of aorta was then washed with distilled water for three times and scanned with a table scanner (Epson 1220 Perfection; Epson Co., Tokyo, Japan). The area of atherosclerotic plaque was measured with the aid of a computer image analysing program (Sigma Scan Pro 5.0; SPSS, Inc., Chicago, IL, USA).

\section{Statistics}

Data are expressed as mean values and standard deviations. The group means were statistically analysed using one-way ANOVA and post hoc LSD test on SigmaStat Advisory Statistical Software (SigmaStat version 14.0; SPSS, Inc.). Significance was defined as a $P$ value less than 0.05.

\section{Results}

\section{Body weight and food intake}

The body-weight gain was similar among the five groups (data not shown). Oxidised cholesterol did not affect the weight gain compared with the control hamsters. Food intake ranged 10.9-11.2 g/d per hamster among the five groups. No significant difference in food intake was seen between the non-oxidised and oxidised cholesterol groups. COP intake by the OxC-0.05 and OxC-0.1 groups was 1.2 and $2.4 \mathrm{mg} / \mathrm{d}$ per hamster, respectively.

\section{Serum total cholesterol, triacylglycerols and low-density lipoprotein cholesterol}

Serum TC increased in response to the amount of cholesterol in the diet. As shown in Table 1, both dietary non-oxidised and oxidised cholesterol elevated serum TC in a dose-dependent manner. Compared with non-oxidised cholesterol, oxidised cholesterol was more hypercholesterolaemic (Table 1). To be more specific, serum TC level in the control, C-0.05, $\mathrm{C}-0 \cdot 1$, OxC-0.05 and OxC-0.1 groups was $2 \cdot 85,3 \cdot 52,4 \cdot 30$, 4.32 and $4.82 \mathrm{mmol} / \mathrm{l}$, respectively. Serum TC in the OxC0.05 and $\mathrm{OxC}-0.1$ groups was 22 and $12 \%$ higher, respectively, compared with the corresponding C-0.05 and C-0.1 hamsters $(P<0 \cdot 05)$. Like TC, serum non-HDL-C exhibited similar change in response to dietary non-oxidised and oxidised cholesterol. Oxidised cholesterol had a greater raising effect on non-HDL-C level than non-oxidised cholesterol in the diet. For HDL-C level, no difference was observed between the $\mathrm{C}-0.05$ and $\mathrm{OxC}-0.05$ groups, but the OxC-0.1 group had an HDL-C level significantly higher than the C-0.1 hamsters (Table 1). Both non-oxidised and oxidised cholesterol groups had a greater non-HDL-C:HDL-C ratio than the control. However, no significant difference was observed between the non-oxidised and oxidised cholesterol groups. Serum TAG increased with the amount of dietary

Table 1. Changes in serum total cholesterol (TC), triacylglycerols, HDL-cholesterol (HDL-C) and non-HDL-cholesterol (non$\mathrm{HDL}-\mathrm{C})$ in hamsters fed the control (CON) and the experimental diets containing $0.05 \%$ non-oxidised cholesterol (C-0.05), $0.10 \%$ non-oxidised cholesterol (C-0.1), $0.05 \%$ oxidised cholesterol (OxC-0.05) and $0.10 \%$ oxidised cholesterol (OxC-0.1) for 6 weeks

(Mean values and standard deviations)

\begin{tabular}{|c|c|c|c|c|c|c|c|c|c|c|}
\hline \multirow[t]{2}{*}{ Diet... } & \multicolumn{2}{|c|}{ CON } & \multicolumn{2}{|c|}{ C-0.05 } & \multicolumn{2}{|c|}{ OxC-0.05 } & \multicolumn{2}{|c|}{$C-0.1$} & \multicolumn{2}{|c|}{$\mathrm{OxC}-0 \cdot 1$} \\
\hline & Mean & SD & Mean & SD & Mean & SD & Mean & SD & Mean & SD \\
\hline $\mathrm{TC}(\mathrm{mmol} / \mathrm{l})$ & $2 \cdot 85^{d}$ & 0.30 & $3.52^{c}$ & 0.31 & $4 \cdot 30^{\mathrm{b}}$ & 0.31 & $4 \cdot 32^{b}$ & 0.45 & $4 \cdot 82^{\mathrm{a}}$ & 0.38 \\
\hline TAG $(\mathrm{mmol} / \mathrm{l})$ & $2 \cdot 61^{\mathrm{e}}$ & 0.30 & $2.90^{d}$ & 0.21 & $3 \cdot 37^{b}$ & 0.27 & $3 \cdot 14^{c}$ & 0.18 & $3.66^{a}$ & 0.26 \\
\hline HDL-C (mmol/l) & $1.52^{c}$ & 0.20 & $1.60^{\mathrm{c}}$ & 0.21 & $1.89^{b}$ & 0.17 & $1.93^{b}$ & 0.19 & $2 \cdot 10^{\mathrm{a}}$ & 0.15 \\
\hline Non-HDL-C (mmol/l) & $1 \cdot 34^{d}$ & 0.16 & $1.93^{\mathrm{c}}$ & 0.34 & $2.42^{\mathrm{b}}$ & 0.30 & $2 \cdot 40^{\mathrm{b}}$ & 0.26 & $2 \cdot 72^{\mathrm{a}}$ & 0.30 \\
\hline Non-HDL-C:HDL-C & $0.88^{b}$ & 0.12 & $1 \cdot 21^{a}$ & 0.18 & $1 \cdot 28^{\mathrm{a}}$ & 0.13 & $1 \cdot 24^{\mathrm{a}}$ & 0.15 & $1 \cdot 29^{a}$ & 0.21 \\
\hline
\end{tabular}

$\mathrm{a}, \mathrm{b}, \mathrm{c}, \mathrm{d}, \mathrm{e}$ Mean values within a row with unlike superscript letters are significantly different $(P<0.05)$. 
non-oxidised and oxidised cholesterol in a dose-dependent manner. However, it was demonstrated that the oxidised cholesterol groups had higher serum TAG levels than the corresponding hamsters maintained on the non-oxidised cholesterol diet (Table 1).

\section{Liver cholesterol and cholesterol oxidation products}

The amount of cholesterol in the liver increased with both non-oxidised and oxidised cholesterol in the diets in a dosedependent manner (Table 2). There was no difference in hepatic cholesterol between the $\mathrm{C}-0.05$ and $\mathrm{OxC}-0.05$ groups. In contrast, hepatic cholesterol in the $\mathrm{C}-0 \cdot 1$ group was greater than that in $\mathrm{OxC}-0 \cdot 1$ hamsters. The results demonstrated that dietary COP could deposit in the liver, with $7 \alpha$ - hydroxycholesterol and 7 $\beta$-hydroxycholesterol accumulating the most (Table 2). COP in the liver of the OxC-0.05 and OxC-0.1 groups were three to five times greater than that of the corresponding non-oxidised cholesterol groups.

\section{Aortic cholesterol and atherosclerotic plaque}

Both the non-oxidised and oxidised cholesterol diets increased deposition of cholesterol in the aorta compared with the control diet (Fig. 2). The two groups fed the OxC-0.05 and OxC0.1 diets demonstrated greater deposition of cholesterol in the aorta compared with the corresponding hamsters fed diets containing $0.05 \%$ and $0.1 \%$ non-oxidised cholesterol $(P<0.05)$. Similarly, both groups fed diets containing oxidised cholesterol had a greater area of atherosclerotic plaque than the corresponding groups fed diets containing non-oxidised cholesterol. However, only the OxC-0.1 group had an area of atherosclerotic plaque significantly larger than the $\mathrm{C}-0 \cdot 1$ hamsters $(P<0.05)$.

\section{Aortic relaxation}

The effect of dietary oxidised cholesterol on aortic function was examined by measuring aortic contraction-relaxation ability. The concentration-response curve of phenylephrine-acetylcholine was constructed (Fig. 3). Compared with the control group, both the non-oxidised and oxidised cholesterol groups showed greater inhibition on acetylcholine-induced relaxation. In contrast to the non-oxidised cholesterol groups, only the OxC-0.1 group had inhibition on acetylcholine-induced relaxation significantly greater than the C-0.1 hamsters (Fig. 3).

\section{Faecal neutral and acidic sterols}

Addition of both non-oxidised and oxidised cholesterol into the diet caused greater faecal excretion of both neutral and acidic sterols compared with the control diet (Table 3). However, no difference in faecal excretion of both neutral and acidic sterols was seen between the non-oxidised and oxidised cholesterol groups.

\section{Discussion}

The present results clearly demonstrated that dietary oxidised cholesterol had a greater hypercholesterolaemic activity than cholesterol itself in hamsters. The analysis of lipoprotein profile showed that, accompanied with elevation in serum TC, incorporation of oxidised cholesterol in the diet increased proportionally HDL-C and non-HDL-C levels, thus having no effect on the non-HDL-C:HDL-C ratio, compared with nonoxidised cholesterol in the diet. The Golden Syrian hamster was chosen in the present study because its cholesterol metabolism is similar or close to that in humans ${ }^{20}$. The present result was in agreement with that in an earlier report by Podrez et $a .^{21}$, who found that the rabbits fed a diet containing $5 \%$ of oxidised cholesterol derivatives had a 5-fold increase in serum cholesterol level compared with non-oxidised cholesterol-fed rabbits. In another study in rats, Al Kanhal et al. ${ }^{6}$ examined the toxicity of oxidised cholesterol and found that the addition of $1 \%$ oxidised cholesterol in the diet could significantly elevate serum TC by $8 \%$ greater than dietary $1 \%$ non-oxidised cholesterol, although the authors did not intend to examine the serum cholesterol-raising activity of oxidised cholesterol v. non-oxidised cholesterol. Quantitatively, we observed that oxidised cholesterol elevated serum TC by $12-25 \%$ greater than the corresponding non-oxidised cholesterol. In this regard, greater hypercholesterolaemic activity associated with oxidised cholesterol in hamsters than in rats can be explained by the observation that rats are

Table 2. Changes in liver total cholesterol (TC) and cholesterol oxidation products (COP) in hamsters fed the control (CON) and the experimental diets containing $0.05 \%$ non-oxidised cholesterol (C-0.05), $0.10 \%$ non-oxidised cholesterol (C-0.1), $0.05 \%$ oxidised cholesterol (OxC-0.05) and $0.10 \%$ oxidised cholesterol (OxC-0.1) for 6 weeks

(Mean values and standard deviations)

\begin{tabular}{|c|c|c|c|c|c|c|c|c|c|c|}
\hline \multirow{2}{*}{ Diet. . } & \multicolumn{2}{|c|}{ CON } & \multicolumn{2}{|c|}{ C-0.05 } & \multicolumn{2}{|c|}{ OxC-0.05 } & \multicolumn{2}{|c|}{$\mathrm{C}-0 \cdot 1$} & \multicolumn{2}{|c|}{$\mathrm{OxC}-0.1$} \\
\hline & Mean & SD & Mean & SD & Mean & SD & Mean & SD & Mean & SD \\
\hline $\begin{array}{l}\text { TC }(\mathrm{mg} / \mathrm{g}) \\
\text { COP }(\mu \mathrm{g} / \mathrm{g})\end{array}$ & $6 \cdot 21^{d}$ & 2.09 & $16 \cdot 93^{c}$ & 3.99 & $18 \cdot 31^{\mathrm{c}}$ & $5 \cdot 80$ & $44 \cdot 60^{\mathrm{a}}$ & 5.56 & $23.52^{b}$ & $5 \cdot 07$ \\
\hline $5 \alpha$-Hydroxycholesterol & \multicolumn{2}{|c|}{ ND } & $36.53^{a}$ & 8.02 & $42 \cdot 39^{a}$ & $10 \cdot 54$ & $22 \cdot 33^{b}$ & $3 \cdot 28$ & $37 \cdot 11^{\mathrm{a}}$ & $8 \cdot 24$ \\
\hline $7 \alpha$-Hydroxycholesterol & \multicolumn{2}{|c|}{ ND } & $0.00^{\mathrm{C}}$ & 0.00 & $40.54^{a}$ & $10 \cdot 27$ & $0.80^{\mathrm{b}}$ & 0.19 & $45 \cdot 60^{\mathrm{a}}$ & $9 \cdot 07$ \\
\hline $7 \beta$-Hydroxycholesterol & $0.09^{b}$ & 0.03 & $0 \cdot 12^{b}$ & 0.02 & $46 \cdot 16^{a}$ & 9.05 & $0.11^{b}$ & 0.04 & $43 \cdot 14^{\mathrm{a}}$ & $7 \cdot 04$ \\
\hline$\alpha$-Epoxide & \multicolumn{2}{|c|}{ ND } & $0.24^{b}$ & 0.17 & $0.73^{a}$ & 0.32 & $0.27^{b}$ & 0.06 & $1.03^{\mathrm{a}}$ & 0.29 \\
\hline Total & $0.09^{d}$ & 0.03 & $41.47^{b}$ & 8.21 & $129 \cdot 81^{a}$ & $52 \cdot 20$ & $23.51^{c}$ & 3.57 & $126 \cdot 88^{a}$ & 30.63 \\
\hline
\end{tabular}

ND, not detectable.

a,b,c,d Mean values within a row with unlike superscript letters are significantly different $(P<0.05)$. 
(A)

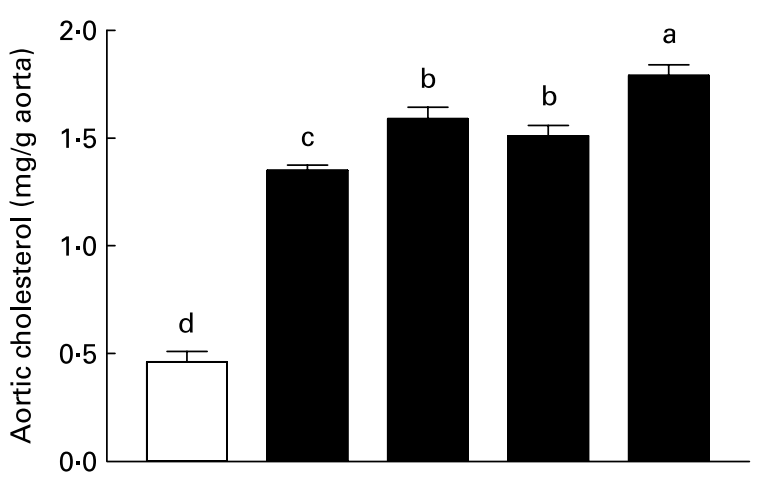

(B)

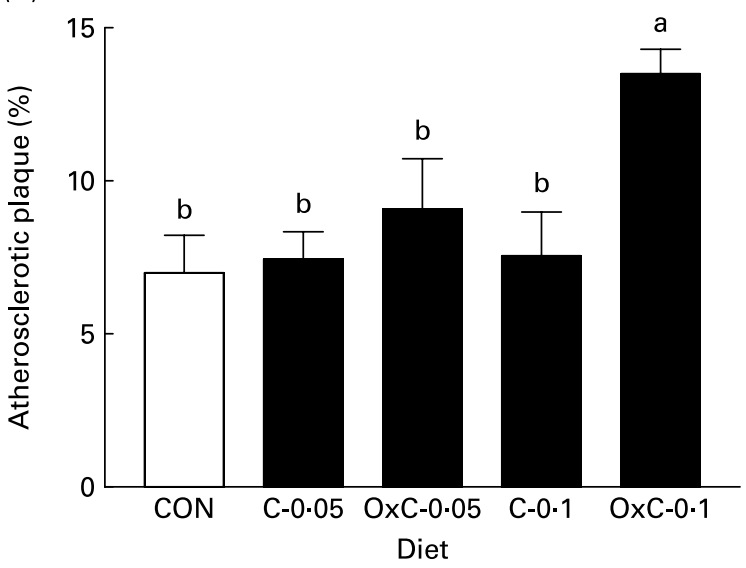

Fig. 2. Effect of dietary oxidised cholesterol on cholesterol content (A) and atherosclerotic plaque $(B)$ in the aorta in hamsters. CON, control diet; C- 0.05 , diet containing $0.05 \%$ non-oxidised cholesterol; C- 0.1 , diet containing $0.1 \%$ non-oxidised cholesterol; OxC-0.05, diet containing $0.05 \%$ oxidised cholesterol mixture; OxC-0.1, diet containing $0.1 \%$ oxidised cholesterol mixture. Values are means (n 10), with standard deviations represented by vertical bars. ${ }^{a, b, c, d}$ Mean values with unlike letters are significantly different $(P<0.05)$.

hyporesponsive and have greater bile acid excretion capacity than hamsters in response to dietary cholesterol loading ${ }^{22}$.

Measurement of both atherosclerotic plaque and aortic cholesterol provides direct evidence that oxidised cholesterol is more atherogenic. The present result showed clearly that dietary oxidised cholesterol significantly increased atherosclerotic plaque and cholesterol content in the aorta compared with dietary non-oxidised cholesterol (Fig. 2). It had been shown that oxidised cholesterol in the diet could be absorbed in the small intestine and incorporated into chylomicrons, VLDL, HDL and LDL in human subjects ${ }^{23,24}$. Similarly, rabbits fed an oxidised cholesterol diet had an increase in fatty streak lesion in the aorta ${ }^{14}$. Oxidised lipoproteins, particularly oxidised LDL, are atherogenic and play a key role in pathogenesis of $\mathrm{CHD}^{23}$. However, the origin of oxidised lipoproteins in vivo is not clear but at least part of circulating oxidised LDL originates from the $\operatorname{diet}^{24}$, deposits in endothelial cells and initiates atherosclerotic plaque $e^{23,24}$. The present results demonstrated that oxidised cholesterol in the diet was not only more hypercholesterolaemic but also more atherogenic compared with cholesterol itself.
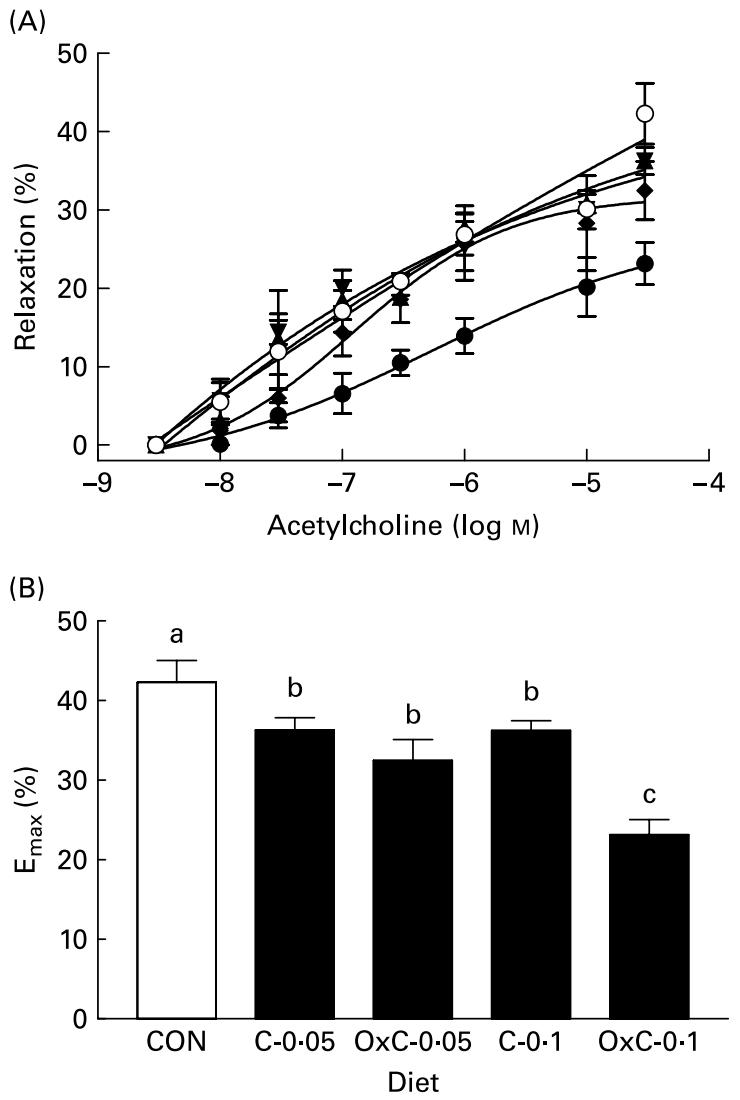

Fig. 3. Effect of dietary oxidised cholesterol on acetylcholine-induced relaxation $(A)$ and maximum relaxation $(B)$ in aorta rings. $(O)$, Control diet; $(\mathbf{\Lambda})$, diet containing $0.05 \%$ non-oxidised cholesterol; $(\mathbf{\nabla})$, diet containing $0.1 \%$ non-oxidised cholesterol; $(\bullet)$, diet containing $0.05 \%$ oxidised cholesterol mixture; $(\bullet)$, diet containing $0.1 \%$ oxidised cholesterol mixture. Values are means $(n 10)$, with standard deviations represented by vertical bars. ${ }^{\mathrm{a}, \mathrm{b}, \mathrm{c}}$ Mean values with unlike letters are significantly different $(P<0.05)$.

No study to date has examined the effect of dietary oxidised cholesterol on functionality of arteries in vivo. The present study found that both non-oxidised and oxidised cholesterols significantly inhibited acetylcholine-induced relaxation of the aorta in hamsters (Fig. 3). However, $0 \cdot 1 \%$ oxidised cholesterol in the diet was more potent in inhibition than non-oxidised cholesterol, suggesting that dietary oxidised cholesterol could cause greater endothelial damage. Endothelium plays a key role in regulating cardiovascular function. The presence of oxidised lipoproteins is believed to be an early event in the pathogenesis of endothelial dysfunction-associated $\mathrm{CVD}^{25,26}$. The present result was in agreement with that of Zhao \& Tackett $^{27}$ and Chan et al. ${ }^{28}$, who found that oxidised LDL significantly inhibited acetylcholine-induced relaxation and increased contractile responses to vasconstrictors when blood vessels were incubated with oxidised LDL. Most likely, dietary oxidised cholesterol could incorporate into lipoproteins and produce oxidised LDL, which accumulated on the endothelium to promote atherosclerotic plaque and impair endothelium-dependent vasorelaxation ${ }^{15,24}$.

Dietary oxidised cholesterol not only increased serum TC but also reduced the liver cholesterol level compared with non-oxidised cholesterol (Table 2). When $0.05 \%$ oxidised 
Table 3. Faecal excretion of neutral and acidic sterols ( $\mathrm{mg} /$ hamster per $\mathrm{d}$ ) in hamsters fed the control (CON) and the experimental diets containing $0.05 \%$ non-oxidised cholesterol (C-0.05), $0.10 \%$ non-oxidised cholesterol (C-0.1), $0.05 \%$ oxidised cholesterol (OxC-0.05) and 0.10\% oxidised cholesterol (OxC-0.1) for 6 weeks

(Mean values and standard deviations)

\begin{tabular}{|c|c|c|c|c|c|c|c|c|c|c|}
\hline \multirow{2}{*}{ Diet... } & \multicolumn{2}{|c|}{ CON } & \multicolumn{2}{|c|}{ C-0.05 } & \multicolumn{2}{|c|}{ OxC-0.05 } & \multicolumn{2}{|c|}{ C-0.1 } & \multicolumn{2}{|c|}{$\mathrm{Ox}-\mathrm{C}-0 \cdot 1$} \\
\hline & Mean & SD & Mean & SD & Mean & SD & Mean & SD & Mean & SD \\
\hline \multicolumn{11}{|l|}{ Neutral sterols } \\
\hline Coprastanol & $1 \cdot 65^{c}$ & 0.53 & $3.51^{a}$ & 0.25 & $2 \cdot 50^{\mathrm{b}}$ & 0.20 & $3 \cdot 82^{a}$ & 0.21 & $3 \cdot 92^{a}$ & 0.48 \\
\hline Coprostanone & $0.08^{b}$ & 0.01 & $0 \cdot 11^{a}$ & 0.03 & $0.09^{a}$ & 0.01 & $0 \cdot 10^{\mathrm{a}}$ & 0.03 & $0 \cdot 13^{a}$ & 0.30 \\
\hline Cholesterol & $0.70^{\mathrm{b}}$ & 0.13 & $1.46^{\mathrm{a}}$ & 0.20 & $1.63^{\mathrm{a}}$ & 0.28 & $1 \cdot 39^{a}$ & 0.12 & $1.44^{\mathrm{a}}$ & 0.22 \\
\hline Dihydrocholesterol & $0.84^{b}$ & 0.16 & $1.03^{a, b}$ & 0.06 & $1 \cdot 16^{\mathrm{a}}$ & 0.17 & $1.02^{a, b}$ & 0.12 & $1 \cdot 15^{\mathrm{a}}$ & 0.06 \\
\hline Campersterol & $0 \cdot 17^{b}$ & 0.03 & $0 \cdot 21^{a, b}$ & 0.02 & $0 \cdot 24^{a}$ & 0.01 & $0 \cdot 24^{a, b}$ & 0.04 & $0 \cdot 26^{a}$ & 0.05 \\
\hline$\beta$-Sitosterol & 0.08 & 0.02 & 0.07 & 0.01 & 0.08 & 0.01 & 0.07 & 0.01 & 0.08 & 0.01 \\
\hline Total neutral sterols & $3.50^{\mathrm{b}}$ & 0.61 & $6 \cdot 39^{a}$ & 0.28 & $5 \cdot 70^{\mathrm{a}}$ & 0.60 & $6 \cdot 62^{\mathrm{a}}$ & 0.51 & $6.95^{\mathrm{a}}$ & 0.71 \\
\hline \multicolumn{11}{|l|}{ Acidic sterols } \\
\hline Lithocholic acid & $4 \cdot 43^{c, d}$ & 0.60 & $3.93^{d}$ & 0.61 & $6 \cdot 05^{a}$ & 0.34 & $5 \cdot 20^{\mathrm{b}, \mathrm{c}}$ & 0.32 & $5 \cdot 68^{a, b}$ & 0.56 \\
\hline Deoxycholic acid & $0.90^{c}$ & 0.21 & $1 \cdot 14^{\mathrm{b}, \mathrm{c}}$ & 0.32 & $1 \cdot 28^{\mathrm{a}, \mathrm{b}, \mathrm{c}}$ & 0.31 & $1 \cdot 73^{\mathrm{a}}$ & $0 \cdot 16$ & $1 \cdot 67^{\mathrm{a}, \mathrm{b}}$ & 0.34 \\
\hline Chenodeoxycholic acid & $1 \cdot 71$ & 0.14 & 1.53 & 0.29 & $1 \cdot 25$ & 0.51 & 1.76 & $0 \cdot 17$ & $1 \cdot 72$ & 0.35 \\
\hline Cholic acid & $0.80^{c}$ & 0.65 & $3 \cdot 73^{a}$ & 0.53 & $2 \cdot 48^{a, b}$ & 0.74 & $2 \cdot 01^{b}$ & 0.44 & $1 \cdot 87^{\mathrm{b}}$ & 0.22 \\
\hline Total acidic sterols & $9 \cdot 15^{\mathrm{b}}$ & $1 \cdot 13$ & $12 \cdot 04^{a}$ & 1.44 & $12 \cdot 90^{\mathrm{a}}$ & 0.66 & $12 \cdot 49^{\mathrm{a}}$ & 0.81 & $12 \cdot 64^{a}$ & 0.53 \\
\hline
\end{tabular}

a,b,c,d Mean values within a row with unlike superscript letters are significantly different $(P<0.05)$.

cholesterol was added in the diet, no difference was observed in liver cholesterol content between the oxidised cholesterol and non-oxidised cholesterol groups. However, OxC-0.1 hamsters accumulated hepatic cholesterol significantly less than the C- $0 \cdot 1$ hamsters. In this regard, it is known that oxidised cholesterol is much more potent than non-oxidised cholesterol in the inhibition of 3-hydroxy-3-methyl-glutaryl$\mathrm{CoA}$ reductase in human fibroblasts ${ }^{29}$. Individual oxidised cholesterol derivates have been studied with regard to their potency in inhibiting 3-hydroxy-3-methyl-glutaryl-CoA reductase in a large number of mammalian cells ${ }^{30}$, finding that half-maximal inhibitory concentration $\left(\mathrm{IC}_{50}\right)$ ranged from $0 \cdot 1$ to $25 \mu \mathrm{M}$. In addition, reduction in 3-hydroxy-3-methyl-glutaryl-CoA reductase activity associated with oxidised cholesterol feeding compared with non-oxidised cholesterol was also seen in hens ${ }^{31}$. In the present study, dietary oxidised cholesterol led to the accumulation of COP in the liver (Table 2). On one hand, stronger inhibition on 3-hydroxy-3-methyl-glutaryl-CoA reductase by $\mathrm{COP}$ in hamsters fed the OxC-0.1 diet could reduce cholesterogenesis in the liver, leading to lesser cholesterol accumulation in the liver than that in hamsters fed the corresponding non-oxidised cholesterol diet. On the other hand, feeding oxidised cholesterol may not necessarily decrease the secretion of hepatic VLDL and instead it may increase its secretion, as one report demonstrated that VLDL secretion by hepatocytes from oxidised cholesterol-fed rabbits was dramatically increased in comparison with those from non-oxidised cholesterol-fed rabbits ${ }^{21}$.

Greater potency in elevating serum TC associated with the oxidised cholesterol feeding compared with non-oxidised cholesterol in the diet suggested a shift in balance among dietary intake, synthesis and catabolism. On one hand, the removal of LDL-cholesterol is mainly mediated by LDL receptors in the liver. Although the present study did not provide direct evidence that dietary oxidised cholesterol inhibited LDL receptors, several studies on cell lines have demonstrated that most COP caused suppression on levels of ${ }^{125} \mathrm{I}$-labelled LDL binding in human fibroblasts ${ }^{29}$, mouse teratocarcinoma cells $^{32}$ and human epithelioid carcinoma cells ${ }^{33}$. Inhibition on LDL receptor activity was reported to be associated with a decrease in mRNA level of LDL receptors in HepG2 cells ${ }^{34}$. It was most likely that dietary oxidised cholesterol caused a relative decrease in LDL receptor activity, led to an inefficient removal of LDL-cholesterol from the circulation and serum TC was thus raised ${ }^{29}$. On the other hand, although it is speculative, dietary COP may increase plasma oxysterols, induce up regulation of scavenger B receptor 36 in monocytes/ macrophages, result in an increased uptake of modified LDL and thus lead to an ensuing increase in both vascular cholesterol and $\mathrm{COP}^{30,35}$. In fact, the present study found that oxidised cholesterol feeding led to greater accumulation of cholesterol and atherosclerosis plaque in the aorta (Fig. 2).

The current recommendation for daily cholesterol intake is less than $300 \mathrm{mg}$, and in some countries the food label must present the amount of cholesterol in foods because cholesterol increases plasma TC and is associated with the risk of CHD. Of significance, if the data in the present study can be applied to humans, with human diets containing not only cholesterol but also oxidised cholesterol, the present study emphasises that the oxidised cholesterol is much more hypercholesterolaemic.

\section{Acknowledgements}

We thank the Hong Kong Research Grant Council for supporting this research (project no. CUHK 4586/06M).

\section{References}

1. Kumar N \& Shingal OP (1992) Effect of processing conditions on the oxidation of cholesterol in ghee. J Sci Food Agric 58, 267-273.

2. Escharte M, Zulet MA \& Astiasaran I (2001) Oxidation process affecting fatty acids and cholesterol in fried and roasted salmon. J Agric Food Chem 49, 5662-5667. 
3. Smith L (1987) Cholesterol autoxidation. Chem Phys Lipids 44, $87-125$.

4. Osada K, Komada T, Cui L, Yamada K \& Sugano M (1993) Level and formation of oxidized cholesterols in processed marine foods. J Agric Food Chem 41, 1893-1898.

5. Tai CY, Chen YC \& Chen BH (2000) Analysis, formation and inhibition of cholesterol oxidation products in foods: an overview (part II). J Food Drug Anal 8, 1-15.

6. Al Kanhal MA, Ahmad F, Al Othman AA, Al Orf S \& Al Murshed KS (2002) Effect of pure and oxidized cholesterolrich diets on some biochemical parameters in rats. Int $J$ Food Sci Nutr 53, 381-388.

7. Homma Y, Kondo Y, Kaneko M, Kitamura T, Nyou WT, Yanagisawa M, Yamamoto Y \& Katizoe T (2004) Promotion of carcinogenesis and oxidative stress by dietary cholesterol in rat prostate. Carcinogenesis 25, 1011-1014.

8. Wrensch MR, Petrakis NL, Grenke LD, Miike R, Ernster VL, King EB, Hauck WW, Craig JC \& Goodson WH (1989) Breast fluid cholesterol and cholesterol $\beta$-epoxide concentration in women with benign breast disease. Cancer Res 49, 2168-2174.

9. Tomoyori H, Carvajal O, Nakayama M, Kishi T, Sato M, Ikeda I \& Imaizumi K (2002) Lymphatic transport of dietary cholesterol oxidation products, cholesterol and triacylglycerols in rats. Biosci Biotechnol Biochem 66, 828-834.

10. Emanuel H, Hassel CA, Addis PB, Bergmann SD \& Zavoral JH (1991) Plasma cholesterol oxidation products (oxysterols) in human subjects fed a meal rich in oxysterols. J Food Sci 56, 843-847.

11. Seillan C (1990) Oxysterol mediated changes in fatty acid distribution and lipid synthesis in cultured bovine aortic smooth muscle cells. Lipids 25, 172-176.

12. Massey JB (2006) Membrane and protein interactions of oxysterols. Curr Opin Lipidol 17, 296-301.

13. van Reyk DM, Brown AJ, Hult'en LM, Dean RT \& Jessup W (2006) Oxysterols in biological systems: source, metabolism and pathophysiological relevance. Redox Rep 11, 252-262.

14. Staprans H, Pan XM, Joseph HR \& Feingold KR (1998) Oxidized cholesterol in the diet accelerates the development of aortic atherosclerosis in cholesterol-fed rabbits. Arterioscler Thromb Vasc Biol 18, 977-983.

15. Staprans H, Pan XM, Joseph HR, Grunfeld C \& Feingold KR (2000) Oxidized cholesterol in the diet accelerates the development of aortic atherosclerosis in LDL-receptor and apolipoprotein E-deficient mice. Arterioscler Thromb Vasc Biol 20, $708-714$.

16. Sanders TA \& Sandaradura S (1992) The cholesterol-raising effect of coffee in the Syrian hamster. Br J Nutr 68, 431-434.

17. Chan PT, Fong WP, Cheung YL, Huang Y, Ho WKK \& Chen ZY (1999) Jasmine green tea epicatechins are hypolipidemic in hamsters fed a high fat diet. J Nutr 129, 1094-1101.

18. Leung HS, Leng FP, Yao X, Ko WH, Chen ZY, Vanhoutte PM \& Huang Y (2006) Endothelial mediators of the acetylcholineinduced relaxation of the rat femoral artery. Vascular Pharmacol 44, 299-308.

19. Zhang ZS, James AE, Huang Y, Ho WKK, Sahota S \& Chen ZY (2005) Quantification and characterization of aortic cholesterol in rabbits fed a high-cholesterol diet. Int J Food Sci Nutr 56, 359-366.

20. Sujiyama $\mathrm{Y}$, Odaka $\mathrm{H}$, Itokawa $\mathrm{S}$, Ishikawa $\mathrm{E}$, Tomari $\mathrm{Y}$ \& Ikeda H (1995) TMP-153, a novel ACAT inhibitor, lowers plasma cholesterol through its hepatic action in golden hamsters. Atherosclerosis 118, 145-152.

21. Podrez EA, Kosykh VA, Lankin VZ, Novikov DK, Volgushev SA, Viktorov AV \& Rapin VS (1989) Secretion of very lowdensity lipoproteins by cultured rabbits hepatocytes with hypercholesteremia, induced by purified cholesterol and containing antooxidation products. Biokimiia 54, 999-1008.

22. Horton JD, Cuthbert JA \& Spady DK (1995) Regulation of hepatic $7 \alpha$-hydroxylase expression and response to dietary cholesterol in the rat and hamster. $J$ Biol Chem 270, 5381-5387.

23. Fraley AE \& Tsimilas S (2006) Clinical applications of circulating oxidized low-density lipoprotein biomarkers in cardiovascular disease. Curr Opin Lipidol 17, 502-509.

24. Staprans I, Pan XM, Rapp JH \& Feingold KH (2005) The role of dietary oxidized cholesterol and oxidized fatty acids in the development of atherosclerosis. Mol Nutr Food Res 49, $1075-1082$.

25. Gutierrez J, Ballinger SW, Darley-Usmar VM \& Landar A (2006) Free radicals, mitochondria, and oxidized lipids: the emerging role in signal transduction in vascular cells. Circ Res 99, 924-932.

26. Ryoo S, Lemmon CA, Gupta G, White AR, Nyhan D, Shoukas A, Romer LH \& Berkowitz DE (2006) Oxidized low-density lipoprotein-dependent endothelial arginase II activation contributes to impaired nitric oxide signaling. Circ Res 99, 951-960.

27. Zhao L \& Tackett RL (1998) Oxidized low-density lipoprotein inhibits acetylcholine-induced vasorelaxation and increases 5-HT-induced vasoconstriction in isolated human saphenous vein. J Pharmacol Exp Ther 284, 637-643.

28. Chan H, Lougheed M, Laher I \& Steinbrecher UP (2003) Oxidized low-density lipoprotein inhibits endothelium-dependent vasodilation by an antioxidant-sensitive, lysophosphatidylcholine-independent mechanism. $J$ Cardiovasc Pharmacol 41, $856-865$.

29. Brown MS \& Goldstein IL (1974) Suppression of 3-hydroxy-3methyl-glutaryl coenzyme A reductase activity and inhibition of growth of human fibroblasts by 7-ketocholesterol. J Biol Chem 249, 7306-7314.

30. Schroepfer GF Jr (2000) Oxysterols: modulators of cholesterol metabolism and other processes. Physiol Rev 80, 361-554.

31. Naber EC, Allred JB, Winget CJ \& Stock AE (1985) Effect of cholesterol oxidation products on cholesterol metabolism in the lay hen. Poult Sci 64, 675-680.

32. Goldstein JL, Brown MS, Krieger M, Anderson RGW \& Mintz B (1979) Demonstration of low-density lipoprotein receptors in mouse teratocarnoma stem cells and description of a method for producing receptor-deficient mutant mice. Proc Natl Acad Sci U $S$ A 76, 2843-2847.

33. Schneider WJ, Basu SK, McPhaul MJ, Golstein JL \& Brown MS (1979) Solubilization of the low-density lipoprotein receptor. Proc Natl Acad Sci U S A 76, 5577-5581.

34. Srivastava RAK, Ito K, Hess M, Srivastava N \& Schonfeld G (1995) Regulation of low-density lipoprotein receptor gene expression in HepG2 and Caco2 cells by palmitatem oleate, and 25-hydroxycholesterol. J Lipid Res 36, 1434-1446.

35. Nicholson AC, Han J, Febbraio M, Silversterin RL \& Hajjar DP (2001) Role of CD36, the macrophage class B scavenger receptor in atherosclerosis. Ann N Y Acad Sci 947, 224-228. 